\title{
Fluctuations and correlations in the polarization patterns of a Kerr medium
}

\author{
Miguel Hoyuelos, Pere Colet, and Maxi San Miguel \\ Instituto Mediterráneo de Estudios Avanzados, IMEDEA* (CSIC-UIB), Campus Universitat Illes Balears, \\ E-07071 Palma de Mallorca, Spain
}

(Received 22 December 1997)

\begin{abstract}
We study correlations among different components of the spectrum of the light intensity field close to a pattern forming instability associated with the polarization of the light field. In particular we find strong correlations between opposite wave vectors of critical wave number and anticorrelations of the zero wave number and the critical one. These anticorrelations are a manifestation of nonlinear critical fluctuations of the polarization of light. [S1063-651X(98)03406-0]
\end{abstract}

PACS number(s): 05.40.+j, 47.54.+r, 42.65.Sf

\section{INTRODUCTION}

It is well known that close to an instability point fluctuations become large, as they do, for example, close to a critical point in an equilibrium phase transition. In an instability leading to pattern formation, the spectrum of fluctuations below the instability threshold is peaked around a wave number associated with the spatial periodicity of the pattern that is formed above threshold. In this way the spectrum of fluctuations is a noisy precursor [1] that identifies a preferred wave number and anticipates the above-threshold pattern. This situation has been studied in detail in experiments in fluid dynamics [2] where thermal fluctuations are observed below the onset of thermal convection. The fluctuating power spectrum has been used to characterize the selected wave number and for a quantitative measurement of the strength of thermal fluctuations.

A basic wave-vector selection process is of linear nature and, for an isotropic system, determines the modulus of the wave vector of fastest growth. This is reflected in a ring of maximum power in the below-threshold spectrum. The selection of a discrete set of wave vectors within this ring is a nonlinear process in which the correlations among the wave vectors with same modulus play a definite role. The discrete set of selected modes determine the pattern that is formed above threshold. Such correlations among the linearly selected wave vectors, which, to our knowledge, have not been analyzed previously in fluid systems, have been considered recently [3] in pattern-forming nonlinear optical systems $[4,5]$. Correlations can be understood here in terms of a process involving the simultaneous emission of twin photons. In addition, these systems present some important peculiarities that make the study of these correlations particularly interesting. First, the power spectrum of fluctuations below threshold is easily observed as the far-field intensity pattern. Second, the fluctuations have contributions of quantum origin (quantum noise) and the observed correlations can encode specific features of quantum statistics. Third, patterns here are spatial structures of a light field and light has a vectorial character that very naturally leads to the study of vectorial correlations. This vectorial character is associated

\footnotetext{
*World Wide Web address: http://www.imedea.uib.es/PhysDept/
}

with the polarization of light, which is an additional degree of freedom leading to polarization patterns.

In this paper we analyze such correlations close to an instability leading to a polarization pattern. Specifically we consider an optical cavity filled with a nonlinear optical material, in this case an isotropic Kerr medium. The cavity is pumped with an external linearly polarized input field (which we take to be $\hat{x}$ polarized). This situation is described by a vectorial version of the scalar model of Lugiato and coworkers $[6,7]$ in which a Turing optical instability was described. The generalization to account for the vectorial degree of freedom of light was described by Geddes et al. [8]. For a self-defocusing medium no pattern-forming instability occurs when neglecting the vectorial character of the field [9]. However, in the vector model a roll $\hat{y}$-polarized pattern emerges, beyond an instability, on top of an $\hat{x}$-polarized homogeneous background [8]. We find two types of strong correlations that can be physically understood in terms of individual four-wave-mixing processes. (a) Among the wave vectors with linearly selected wave number, a maximum correlation between opposite vectors occurs. This is a linear phenomenon that can be found outside the critical region of fluctuations. It is related to symmetry breaking by the pump field. (b) An anticorrelation is found between the zero wave vector of the spectrum of fluctuations (associated with the $\hat{x}$-polarized component) and the ring of linearly selected wave vectors (associated with the $\hat{y}$-polarized component). This anticorrelation is a manifestation of nonlinear critical fluctuations and therefore only observed very close to threshold. We also study these two types of correlations above threshold, showing that indeed the correlations below threshold anticipate properties found in the pattern that emerges above threshold.

Our results here are obtained within a semiclassical approach in which specific features of quantum statistics are neglected. The obtained anticorrelations between the two polarization components of the light field in the patternformation process open the way to the search for quantum noise aspects of these anticorrelations [10].

\section{DESCRIPTION OF THE MODEL}

The dynamics of the electric field inside an optical cavity with a Kerr medium can be described, in the mean-field ap- 
proximation, by two equations for the independent components of the scaled slowly varying amplitude of the field $[8,11]$,

$$
\begin{aligned}
\frac{\partial E_{ \pm}}{\partial t}= & -(1+i \eta \theta) E_{ \pm}+i a \nabla^{2} E_{ \pm}+E_{0}+i \eta\left[A\left|E_{ \pm}\right|^{2}\right. \\
& \left.+(A+B)\left|E_{\mp}\right|^{2}\right] E_{ \pm},
\end{aligned}
$$

where $E_{ \pm}$are the right and left circularly polarized components of the field, $E_{0}$ is an $\hat{x}$ linearly polarized input field, $\eta=+1(-1)$ corresponds to a self-focusing (self-defocusing) medium, $\theta$ is the cavity detuning, $a$ represents the strength of diffraction, and $\nabla^{2}$ is the transverse Laplacian. $A$ and $B$ are parameters related to the components of the susceptibility tensor $\chi$. We consider an isotropic medium for which $A$ $+B / 2=1 \quad(B \leqslant 2)$. The circularly polarized components of the field are expressed in terms of the $\hat{x}$ and $\hat{y}$ linearly polarized components $E_{x}$ and $E_{y}$ as

$$
E_{ \pm}=\left(E_{x} \pm i E_{y}\right) / \sqrt{2} \text {. }
$$

Equation (1) has an $\hat{x}$-polarized homogeneous symmetric solution in which $E_{s+}=E_{s-}=E_{s}$,

$$
E_{0}=E_{s}\left[1-i \eta\left(2 I_{s}-\theta\right)\right],
$$

where $I_{s}=\left|E_{s}\right|^{2}$. It is well known that the homogeneous solution (3) presents bistability for $\theta>\sqrt{3}$. We will restrict ourselves here to the nonbistable regime $\theta<\sqrt{3}$. We note that Eq. (1) has also an asymmetric solution $\left(E_{s+} \neq E_{s-}\right)$. We will not consider the asymmetric solution in this work because it is only relevant for high values of the input field, above the first instability threshold of the symmetric solution [11]. Here we are concerned with correlations close to this instability threshold.

To analyze the stability of the homogeneous steady state $E_{s}$, as well as the effect of the fluctuations in this state, we consider perturbations of the form

$$
E_{ \pm}=E_{s}+\psi_{ \pm}
$$

in Eq. (1). The linear stability analysis $[8,11]$ shows that there are two modes that can become unstable: a symmetric mode $(S)$ with $\psi_{+}=\psi_{-}$and an asymmetric mode $(A)$ with $\psi_{+}=-\psi_{-}$. In the self-focusing case the mode that becomes unstable is the symmetric one. As the unstable mode has the same polarization as the input field, this situation can be described with a scalar model [6,7]. In two-transversedimensional systems this instability leads to an hexagonal stationary pattern. Here we are interested in the instability of the asymmetric mode that occurs in the self-defocusing case. This instability leads to the growth of the component of the field orthogonally polarized to the driving field $[8,11]$. In the linear regime one finds

$$
E_{ \pm}=E_{s} \pm \psi
$$

and

$$
\begin{gathered}
E_{x}=\sqrt{2} E_{s}, \\
E_{y}=-i \sqrt{2} \psi .
\end{gathered}
$$
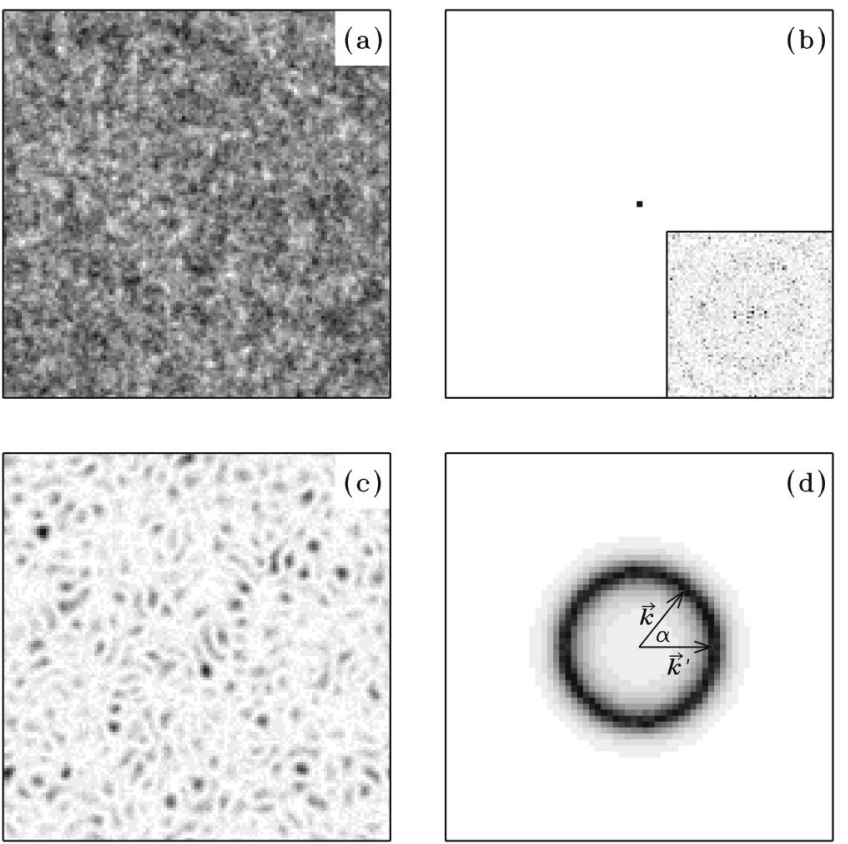

FIG. 1. Near- and far-field intensities below threshold (the farfield intensities correspond to the squared absolute value of the Fourier transform of the electrical field): (a) $\hat{x}$-polarized near-field intensity $I_{x}(\vec{x})=\left|E_{x}(\vec{x})\right|^{2}$, (b) $\hat{x}$-polarized far-field intensity $I_{x}(\vec{k})$ $=\left|E_{x}(\vec{k})\right|^{2}$ [the inset shows $I_{x}(\vec{k})$ after substracting the homogeneous mode contribution], (c) $\hat{y}$-polarized near-field intensity $I_{y}(\vec{x})$, and (d) $\hat{y}$-polarized far-field intensity $I_{y}(\vec{k})$. The parameters are $I_{s}$ $=0.98 I_{s}^{c}$ (below threshold), $a=1, \theta=1, \eta=-1, \epsilon^{2}=10^{-5}$, and $B=3 / 2 . I_{x}(\vec{x})$ and $I_{y}(\vec{x})$ were averaged over 100 samples separated $\Delta t=0.02$ to reduce noise. $I_{y}(\vec{k})$ was averaged over 40000 samples separated $\Delta t=0.5$.

The instability threshold is located at $I_{s}^{c}=1 / B$ and the instability occurs at a critical wave number $k_{c}$,

$$
\begin{gathered}
k_{c}=\sqrt{(\theta+1-2 / B) / a} \text { for } \theta>2 / B-1, \\
k_{c}=0 \text { for } \theta \leqslant 2 / B-1 .
\end{gathered}
$$

For $\theta>2 / B-1$ a stationary striped pattern emerges in the $\hat{y}$ polarized component, while the $\hat{x}$ component remains homogeneous. Above this instability we find, therefore, for the total field, an elliptically polarized pattern with spatially periodic ellipticity. From an experimental point of view an interesting aspect of this situation is that it is possible to separate the finite-wave-number component of the field (pattern) from the homogeneous part by simply using a polarizer.

\section{CORRELATIONS BELOW THRESHOLD}

In order to study correlations below the threshold of pattern formation we need to introduce sources of noise in our description. We follow here the approach already used to study fluctuations in the one-dimensional scalar version of this problem [12]: In a semiclassical description of the problem we can represent different sources of fluctuations by adding Gaussian white noise $\xi_{ \pm}(\vec{x}, t)$ with zero mean and correlations to the right-hand side of Eq. (1), 

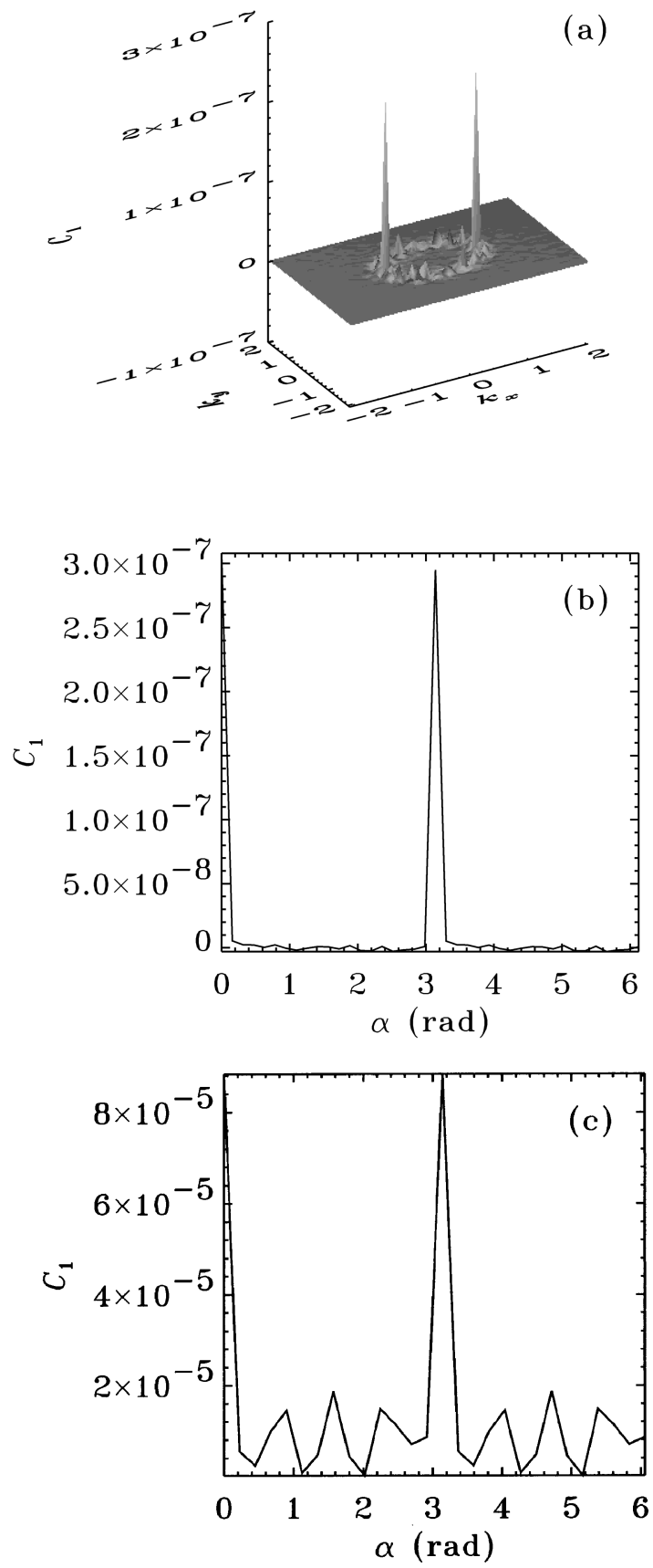

FIG. 2. Correlation $C_{1}$, [see Eq. (9)] (a) as a function of $k_{x}$ and $k_{y}$ for $I_{s}=0.98 I_{s}^{c}$, (b) calculated along the ring $k=k_{c}$ as a function of $\alpha$ for $I_{s}=0.98 I_{s}^{c}$, and (c) versus $\alpha$ for $I_{s}=0.999 I_{s}^{c}$. The rest of the parameters are the same as in Fig. 1. For these parameters $k_{c}$ $=0.82$. The results were obtained after averaging over 40000 samples separated $\Delta t=0.5$.

$$
\begin{gathered}
\left\langle\xi_{i}(\vec{x}, t) \xi_{j}^{*}\left(\vec{x}^{\prime}, t^{\prime}\right)\right\rangle=2 \epsilon^{2} \delta_{i j} \delta\left(\vec{x}-\vec{x}^{\prime}\right) \delta\left(t-t^{\prime}\right), \\
\left\langle\xi_{i}(\vec{x}, t) \xi_{j}\left(\vec{x}^{\prime}, t^{\prime}\right)\right\rangle=0,
\end{gathered}
$$

where the subindices $i, j$ stand for the circularly polarized components \pm .

Slightly below threshold noise excites fluctuations of all wave numbers. However, the wave vector $k_{c}$, which is going to become unstable at threshold, is much less damped than the rest of the modes and, as a consequence, is singled out in the power spectrum of the field. Because a pattern is not yet formed, the excited wave vectors have random directions. In the far field there is a ring of higher intensity with a radius equal to $k_{c}$. In Fig. 1 we show the far-field intensities $I_{x}(\vec{k})$ and $I_{y}(\vec{k})$, corresponding to the spatial power spectrum of the two components $E_{x}(\vec{x})$ and $E_{y}(\vec{x})$ of the field distribution, slightly below the threshold of instability of mode $A$. We also show a snapshot of the spatial configurations of the two components of the electric field intensity (near field) [13]. The far field of the $\hat{x}$-polarized component has a predominant peak at zero wave number corresponding to the homogeneous component of $E_{x}(\vec{x})$. To show the fluctuations around this homogeneous state we have plotted in the inset in Fig. 1(b) the spatial power spectrum of the field distribution after substracting the homogeneous component of $E_{x}(\vec{x})$. The $\hat{y}$-polarized component of the far field displays the characteristic ring shown in Fig. 1(d). The near field of the $\hat{x}$ component shows a noisy homogeneous pattern. The $\hat{y}$ component in the near field is also homogeneous if a long time average is taken. A configuration at a fixed time shows a disordered structure. This structure reveals a characteristic less-stable wave number $k_{c}$ when averaged, for noise reduction, over a short time interval, as done in Fig. 1(c).

We next analyze the correlations among the wave vectors in the ring of the $\hat{y}$-polarized far field. We fix one of these vectors by choosing the point $\vec{k}^{\prime}=\left(k_{c}, 0\right)$ in the plot of Fig. 1 and calculate its correlation with any other wave vector. This is given by the correlation function

$$
C_{1}(\vec{k})=\left\langle\delta I_{y}\left(\vec{k}^{\prime}\right) \delta I_{y}(\vec{k})\right\rangle
$$

where $\delta I_{y}(\vec{k})$ is the fluctuation of the intensity, $\delta I_{y}(\vec{k})$ $=I_{y}(\vec{k})-\left\langle I_{y}(\vec{k})\right\rangle$, and $I_{y}(\vec{k})=\left|E_{y}(\vec{k})\right|^{2} \quad$ (equivalent expresions hold for the $\hat{x}$ component). Angular brackets stand for time average.

In Fig. 2(a) we plot $C_{1}(\vec{k})$ for a distance to threshold $I_{s}=0.98 I_{s}^{c}$ (same parameters as in Fig. 1). We can see a self-correlation at $\vec{k}=\vec{k}^{\prime}$ and another peak at $\vec{k}=-\vec{k}^{\prime}$. We also calculate the correlation $C_{1}$ along the ring as a function of the angle $\alpha$ between $\vec{k}$ and $\vec{k}^{\prime}$, taking $|\vec{k}|=\left|\vec{k}^{\prime}\right|=k_{c}$. In this case we can select different vectors $\vec{k}^{\prime}$ on the ring, calculate the correlation $C_{1}$ for each one of these vectors, and obtain an average. In Fig. 2(b) we plot $C_{1}$ versus $\alpha$ and the correlation at $\alpha=\pi$ is clearly displayed. As we get closer to the threshold we can expect an enhancement of the correlation since fluctuations become larger. This is indeed the case as we can see in Fig. 2(c), where we plot $C_{1}$ as a function of $\alpha$ for a distance to threshold $I_{s}=0.999 I_{s}^{c}$.

Since we are considering correlations below threshold, we may think that the system is not far from the homogeneous solution and fluctuations could be described within a linear analysis. If we linearize Eq. (1) around the homogeneous solution (3), we find for the $\hat{x}$ and $\hat{y}$ components of the fluctuations $\delta E_{x}$ and $\delta E_{y}$ 


$$
\begin{aligned}
\frac{\partial \delta E_{x}}{\partial t}= & -\left[1+i \eta\left(\theta-2 I_{s}\right)-i a \nabla^{2}\right] \delta E_{x}+i \eta 2 I_{s}\left(\delta E_{x}+\delta E_{x}^{*}\right) \\
& +\xi_{x}(\vec{x}, t), \\
\frac{\partial \delta E_{y}}{\partial t}= & -\left[1+i \eta\left(\theta-2 I_{s}\right)-i a \nabla^{2}\right] \delta E_{y}+i \eta I_{s} B\left(\delta E_{y}^{*}-\delta E_{y}\right) \\
& +\xi_{y}(\vec{x}, t),
\end{aligned}
$$

where $\delta E_{x}=E_{x}-\sqrt{2} E_{s}$ and $\delta E_{y}=E_{y}$. These equations show that $\delta E_{x}$ and $\delta E_{y}$ are linearly uncoupled. The correlations in the far field of $E_{y}$ described above can be understood by considering Eq. (10) for the $\hat{y}$ component in Fourier space,

$$
\begin{aligned}
\frac{\partial \delta E_{y}(\vec{k})}{\partial t}= & -\left[1+i \eta\left(\theta-2 I_{s}\right)+i a k^{2}\right] \delta E_{y}(\vec{k}) \\
& +i \eta I_{s} B\left[\delta E_{y}^{*}(-\vec{k})-\delta E_{y}(\vec{k})\right]+\xi_{y}(\vec{k}, t) .
\end{aligned}
$$

Equation (11) shows a linear correlation between wave vectors $\vec{k}$ and $-\vec{k}$ as found in Figs. 2(a) and 2(b). It is important to understand the origin of such correlation: Mathematically, it comes from the term $i \eta I_{s} B\left[\delta E_{y}^{*}(-\vec{k})-\delta E_{y}(\vec{k})\right]$, which breaks the phase invariance of the equation for the complex amplitude $\delta E_{y}$. This symmetry-breaking term is proportional to $I_{s}$, which is nonzero because of the pump field $E_{0}$. The origin of the correlations in the far field of $E_{y}$ is then traced back to symmetry breaking caused by the pump field, which also breaks the global phase symmetry of Eq. (1). In the absence of this term the linear equations are invariant under rotation of the wave vector $\vec{k}$ and different wave vectors are linearly decoupled. The symmetry-breaking term still preserves a discrete symmetry: It is easily seen from Eq. (11) that $I_{y}(\vec{k})$ and $I_{y}(-\vec{k})$ satisfy the same equation. This explains the numerical finding $C_{1}(\alpha) \simeq C_{1}(\alpha+\pi)$ in Figs. 2(b) and 2(c). Upon sufficient averaging, $C_{1}(\alpha)=0$ for $\alpha$ $\neq 0$ and $\alpha \neq \pi$.

At a microscopic level the correlation between $\vec{k}$ and $-\vec{k}$ can be interpreted as a manifestation of the individual four-wave-mixing process in which there is simultaneous emission of two photons that conserve transverse momentum. In this interpretation these two photons are $\hat{y}$ polarized and originate in the annihilation of two $\hat{x}$-polarized photons of the pump field that have zero transverse wave number. As in the four-wave-mixing process, the number of photons is conserved (which reflects conservation of energy since all the photons have the same frequency); it is then natural to expect an anticorrelation between the homogeneous $\hat{x}$-polarized component of the field and the $\hat{y}$-polarized component. However, Eqs. (10) do not account for this anticorrelation since $\delta E_{x}$ and $\delta E_{y}$ are uncoupled.

In order to investigate this possible anticorrelation in which an increase of $I_{y}$ should be accompanied by a decrease of $I_{x}$, we calculate a second correlation function

$$
C_{2}(k)=\left\langle\delta I_{y}(k) \delta I_{x}(0)\right\rangle .
$$
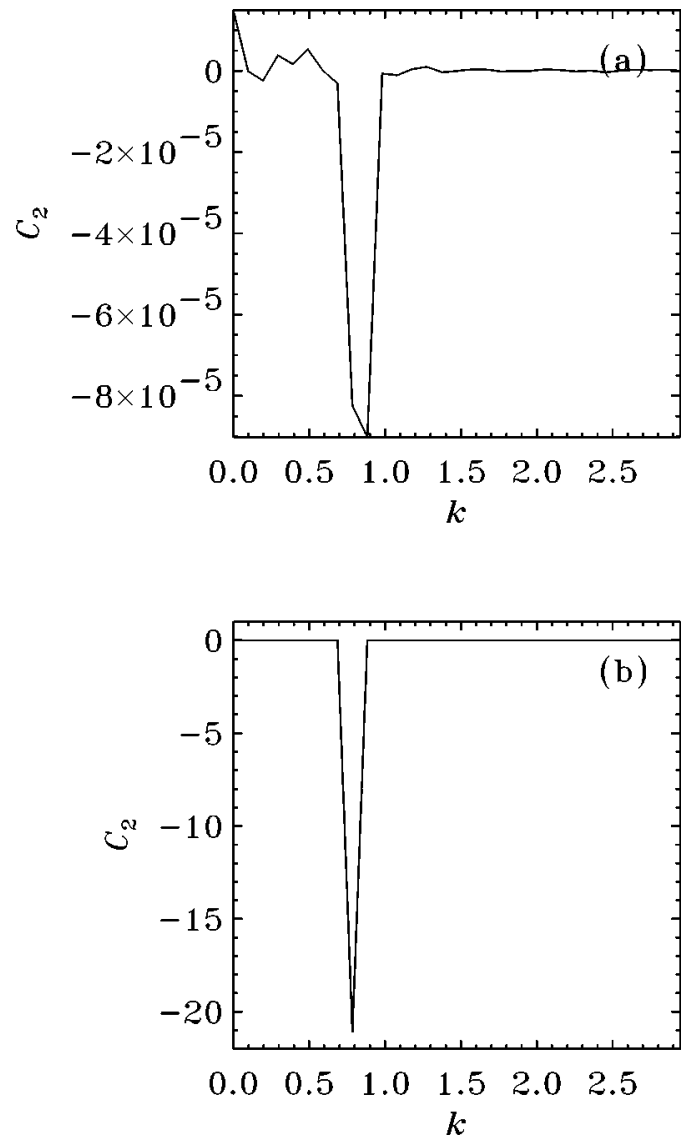

FIG. 3. Correlation $C_{2}(k)$ [see Eq. (12)]. The parameters are the same as in Fig. 1 except for $I_{s}$ : (a) $I_{s}=0.999 I_{s}^{c}$ and (b) $I_{s}$ $=1.05 I_{s}^{c}$ (above threshold). The results were obtained after averaging over 10000 samples separated $\Delta t=0.5$.

$C_{2}(k)$ does not take into account the angle of $\vec{k}$, but it only depends on its modulus $k . \delta I_{x}(0)$ represents the fluctuations of the homogeneous part of the $\hat{x}$ component of the field. $\delta I_{y}(k)$ is the average over orientations of $\vec{k}$ of the fluctuations of $I_{y}$ in Fourier space. Specifically, taking $\quad \vec{k}=(k \cos \phi, k \sin \phi)$, we define $\delta I_{y}(k)$ $=(1 / 2 \pi) \int_{0}^{2 \pi} \delta I_{y}(k \cos \phi, k \sin \phi) d \phi . \delta I_{y}(k)$ at $k=k_{c}$ corresponds to the fluctuations of the $\hat{y}$-polarized precursor of the pattern. If we calculate $C_{2}$ for the same parameters as in Fig. 1 , where the distance to threshold is $I_{s}=0.98 I_{s}^{c}$, we do not obtain any anticorrelation. From our analysis of Eqs. (10), we know that if these anticorrelations exist they have to originate in nonlinear terms. Nonlinearities become important close to the critical point where fluctuations are enhanced. In Fig. 3(a) we plot $C_{2}(k)$ in a situation much closer to threshold, $I_{s}=0.999 I_{s}^{c}$. For these parameters we enter the critical nonlinear regime and, in agreement with our previous reasoning, we now find an anticorrelation at $k=k_{c}$.

In summary, below the threshold for pattern formation there is a linear correlation between the fluctuations of $\vec{k}$ and $-\vec{k}$ in the $\hat{y}$-polarized far field. These fluctuations and correlations become larger in the critical region. In addition, there is a critical nonlinear anticorrelation between the fluctuations of the $\hat{x}$ - and $\hat{y}$-polarized fields that has the same physical origin. 

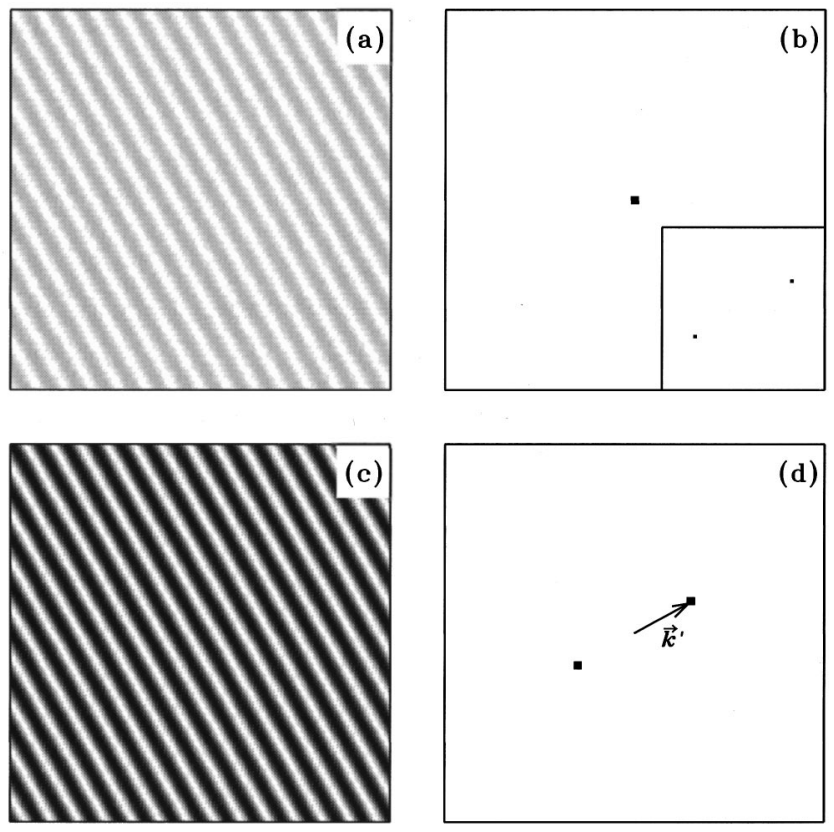

FIG. 4. Near- and far-field intensities above threshold: (a) $I_{x}(\vec{x})$ (near field), (b) $I_{x}(\vec{k})$ (far field) (in the inset the homogeneous mode was substracted to show the contribution to the spectrum of the small-amplitude modulation), (c) $I_{y}(\vec{x})$ (near field), and (d) $I_{y}(\vec{k})$ (far field). The parameters are the same as in Fig. 1, except for $I_{s}$ $=1.05 I_{s}^{c}$. Here it is not necessary to take an average of the nearfield intensities over a short time since the amplitude of the pattern is much larger than the noise amplitude. The far-field intensities are also clearly seen without averaging. The ranges of values for the intensity in the near field are $I_{x}(\vec{x}) \in[1.326,1.356]$ and $I_{y}(\vec{x})$ $\in[0,0.113]$.

\section{CORRELATIONS ABOVE THRESHOLD}

The results discussed in the preceding section are for pumping intensity below the threshold for pattern formation. We now consider the situation above this threshold. We show that the same types of correlations are found. This shows that correlations among the fluctuations below threshold anticipate the features to be observed in the coherent pattern that emerges above threshold. There are two additional reasons for this above-threshold analysis. First, experimental measurements of far-field correlations have already been done above threshold in pattern formation in nonlinear optical systems [5]. Second, as already mentioned, the correlations studied here between the two polarization components of the field in the pattern formation process open the way to search for quantum noise aspects of these correlations. Quantum noise aspects close to an instability have been studied either below or above the instability threshold $[3,14]$.

Above threshold a striped structure is developed in the $\hat{y}$ component of the field (see Fig. 4). In the $\hat{x}$ component also a striped structure appears, produced by nonlinear couplings with the $\hat{y}$ component, but of smaller amplitude; the main contribution to the far field of the $\hat{x}$ component is still the homogeneous mode. The far field of the $\hat{y}$ component has two dots that indicate the arbitrary direction chosen by the system to develop the stripes. It does not have any power at
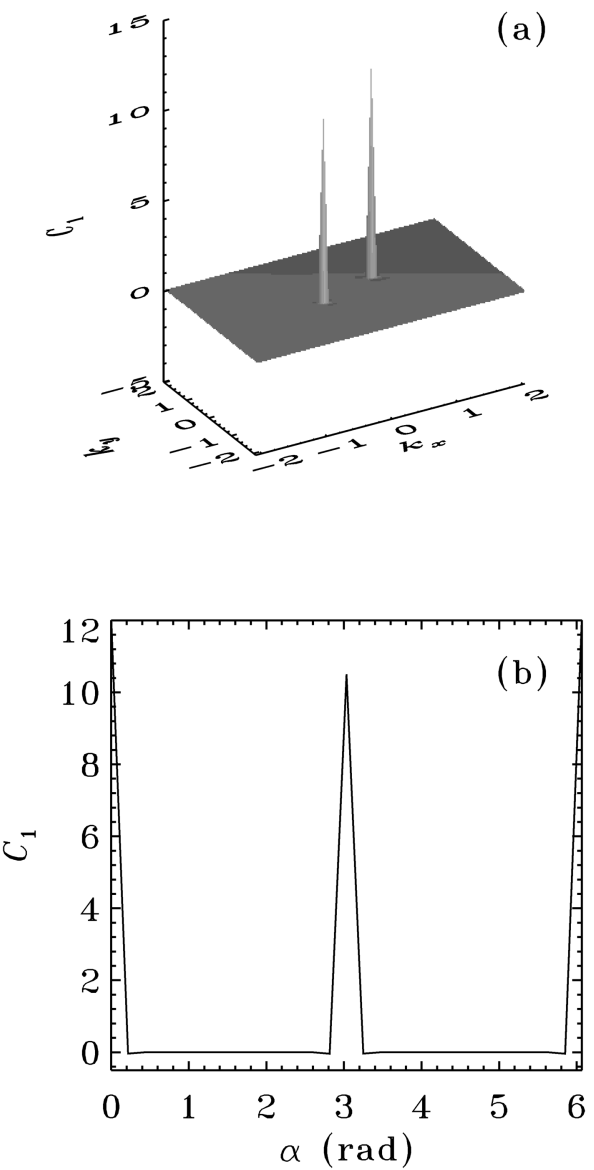

FIG. 5. Correlation $C_{1}$ above threshold [see Eq. (9)] (a) as a function of $k_{x}$ and $k_{y}$ and (b) calculated along the ring $k=k_{c}$. The parameters are the same as in Fig. 1, except for $I_{s}=1.05 I_{s}^{c}$. The results were obtained after averaging over 3000 samples separated $\Delta t=0.5$.

$k=0$ since the $y$ component of the near field $E_{y}(\vec{x})$ has a vanishing mean value.

As in Sec. III, we can calculate the correlation $C_{1}$ between $\delta I_{y}\left(\vec{k}^{\prime}\right)$ and $\delta I_{y}(\vec{k})$ in the whole plane or along a ring of radius $k_{c}$. We choose the vector $\vec{k}^{\prime}$ corresponding to one of the dots in $I_{y}(\vec{k})$, as shown in Fig. 4. Both results are shown in Fig. 5 with results similar to those obtained for the case below threshold. In Fig. 5(a) we see a peak showing the self-correlation at $\vec{k}=\vec{k}^{\prime}$ and another peak at $\vec{k}=-\vec{k}^{\prime}$. In Fig. 5(b) we plot $C_{1}$ as a function of the angle $\alpha$ in the ring of radius $k_{c}$ and we find, as expected, a strong correlation at $\alpha=\pi$. The main difference between Figs. 2 and 5 is that we now have a coherent pattern so that fluctuations in the ring of radius $k_{c}$ are largely suppressed in comparison to the mean value of $I_{y}$ in the two peaks of the power spectrum. These peaks characterize the pattern with a well-defined orientation (see Fig. 1).

We have also calculated the anticorrelation between $I_{x}(0)$ and $I_{y}\left(k_{c}\right)$ above threshold. In this case we calculate $C_{2}$ in the direction determined by the two bright dots in the far field of the $\hat{y}$-polarized intensity $I_{y}(\vec{k})$ (see Fig. 4$)$. The result, shown in Fig. 3(b), displays the anticipated strong anticorrelation. 


\section{ACKNOWLEDGMENTS}

Financial support from the European Union TMR network QSTRUCT (Project No. FMRX-CT96-0077) and from DGICYT Project No. PB94-1167 (Spain) is acknowleged.
We also acknowledge helpful discussions with L. Lugiato. M. H. acknowledges financial support from FOMEC Project No. 290, Departamento de Fisica FCEyN, Universidad Nacional de Mar del Plata, Argentina.
[1] K. Wiesenfeld, J. Stat. Phys. 38, 1071 (1985).

[2] I. Rehberg, S. Rasenat, M. de la Torre Juárez, W. Schöpf, F. Hörner, G. Ahlers, and H. R. Brand, Phys. Rev. Lett. 67, 596 (1991); M. Wu, G. Ahlers, and D. S. Cannell, ibid. 75, 1743 (1995).

[3] A. Gatti, H. Wiedemann, L. A. Lugiato, I. Marzoli, G.-L. Oppo, and S. Barnett, Phys. Rev. A 56, 877 (1997); A. Gatti, L. A. Lugiato, G.-L. Oppo, R. Martin, P. Di Trapani, and A. Berzanskis, Opt. Expr. 1, 21 (1997); L. A. Lugiato, S. Barnett, A. Gatti, I. Marzoli, G.-L. Oppo, and H. Wiedemann, Coherence and Quantum Optics VII (Plenum, New York, 1996), p. 5.

[4] See Chaos Solitons Fractals 4 (8/9) (1994), special issue on nonlinear optical structures, patterns, and chaos, edited by L. A. Lugiato, and references therein; G. Grynberg, A. Maitre, and A. Petrossian, Phys. Rev. Lett. 72, 2379 (1994); T. Ackemann, Y. A. Logvin, A. Heuer, and W. Lange, ibid. 75, 3450 (1995); S. Residori, P. L. Ramazza, E. Pampaloni, S. Boccaletti, and F. T. Arecchi, ibid. 76, 1063 (1996).

[5] A. Maître, A. Petrossian, A. Blouin, M. Pinard, and G. Grynberg, Opt. Commun. 116, 153 (1995).

[6] L. A. Lugiato and R. Lefever, Phys. Rev. Lett. 58, 2209 (1987).
[7] W. J. Firth, A. J. Scroggie, G. S. McDonald, and L. A. Lugiato, Phys. Rev. A 46, R3609 (1992).

[8] J. B. Geddes, J. V. Moloney, E. M. Wright, and W. J. Firth, Opt. Commun. 111, 623 (1994).

[9] In bistable situations, which we do not consider here, pattern formation might still be possible in a defocusing medium.

[10] M. Hoyuelos, P. Colet, A. Sinatra, L. A. Lugiato, and M. San Miguel (unpublished).

[11] M. Hoyuelos, P. Colet, M. San Miguel, and D. Walgraef (unpublished).

[12] M. Aguado, R. F. Rodriguez, and M. San Miguel, Phys. Rev. A 39, 5686 (1989).

[13] These results are obtained by a direct numerical integration of the stochastic Langevin partial differential equations as explained, for example, in M. San Miguel and R. Toral, Instabilities and Nonequilibrium Structures VI (Kluwer Academic, Dordrecht, 1998). Lattices of size $128 \times 128$ were used and the integration steps in time and space were $d t=0.005$ and $d x$ $=0.75$.

[14] L. A. Lugiato and F. Castelli, Phys. Rev. Lett. 68, 3284 (1992); F. Castelli and P. Dotti, Opt. Commun. 113, 237 (1994). 